\title{
Section 22.
}

Design for Subjective Wellbeing 


\title{
Editorial: Design for Subjective Wellbeing
}

\author{
CAIN Rebecca ${ }^{a^{*}}$; PETERMANS Ann ${ }^{b}$; POHLMEYER Annac; DESMET Pieter ${ }^{c}$; OZKARAMANLI Deger ${ }^{d}$ \\ a Loughborough University \\ ${ }^{b}$ Hasselt University \\ c TU Delft \\ d University of Liverpool \\ * Corresponding author e-mail: R.Cain@Iboro.ac.uk
}

\section{Introduction}

SIGWELL is the Design Research Society's Special Interest Group (SIG) focusing on Design for Wellbeing, Happiness and Health. The SIGWELL community has an interest in advancing knowledge, and the development and application of design research in the broadest sense to improve the personal and societal health, wellbeing and happiness of people.

Our SIG is managed by an international board (including the authors here, and in addition - Marc Hassenzahl and Matthias Laschke from the University of Siegen in Germany, Leandro Tonetto from Unisinos, Brazil and and Tiiu Polma from Montreal University in Canada.) Together we represent research in design for subjective wellbeing, experience design, positive design and participatory design across a range of domains from industrial design, engineering, psychology, interaction design and architecture. In 2017 we launched a research manifesto putting forward our vision for our SIG at the World Design Summit in Montreal (Petermans 2017).

\section{From DRS2016 to DRS2018}

In the 2016 conference, the SIGWELL themed tracks included papers on both 'Design for Health' and 'Design for Wellbeing' (Cain et al 2016). Two years ago, we were starting to see more research on subjective wellbeing, and the methods and approaches being reported were highly user-centric, participatory and sometimes critical and speculative. The distilling of design knowledge into frameworks and tools for designers was a popular deliverable of the research.

This year, following the call for tracks, we saw the distinction between the two areas growing, with a large community proposing tracks around design for health(care) and a smaller community proposing tracks around design for wellbeing - particularly subjective wellbeing. Therefore, this year there are two distinct thematic areas for SIGWELL themed sessions at DRS2018, representing the growth and increasing differentiation between Design for Wellbeing (our focus here) and Design for Health (with sessions chaired by Emmanuel Tsekleves). Design for Wellbeing in our context has a particular focus on Subjective Wellbeing, experience design, positive design and design for happiness.

The SIGWELL Design for Wellbeing themed session is an important opportunity to showcase emergent thinking and research from a highly international design community. We see evidence of the growing trend in moving away from designing physical objects and "more stuff" to designing for 
meaningful experiences, where it is the act of designing which can also contribute to a meaningful experience and improved subjective wellbeing rather than the outcome of the design activity per se. Increasingly, the methods to do this are participatory and lead to interesting and timely questions about the role and experiences of co-designers, and the effects of involvement on their own wellbeing.

\section{SIGWELL: Design for Wellbeing papers}

For DRS2018, authors were asked to contribute papers in the general area of subjective wellbeing. Five papers met the quality threshold for the conference and it is interesting to observe that these showed some convergence around two key themes:

Three of the papers used participatory design in a more applied, therapeutic context, focusing on the involvement of people with either specific mental health issues and conditions such as dementia (Garde et al 2018), vulnerable youths (Hong et al 2018) and postnatal depression (Warwick et al 2018). The other two papers played into a more exploratory, theoretical space, looking at how new frameworks and philosophical framing of concepts, e.g. play (Legaard 2018) and somaesthetic practice (Heekyoung and Stahl 2018) could be used to design new meaningful and pleasurable experiences, and so by their nature increase subjective wellbeing.

\subsection{Theme 1: Using participatory design in a therapeutic context -}

Starting with the theme of how involving people with mental health issues in design impacts upon them, Warwick et al (2018) in their paper "Co-designing wellbeing: the commonality of needs between co-designers and mental health service design uses" analysed how the design process impacted on co-designers of a new perinatal mental health service. The co-designers were new mothers, who drew upon their own personal experiences to create a new service 'Mums Matter'. The authors compared these impacts with the eventual service users of the service. Most notably, their paper reveals potential pressure points in the design process and raises important questions about how the risks around these should be mitigated to protect a co-designer's wellbeing. Most pertinently, how should designers' roles expand to explicitly manage a co-designer's wellbeing during the design process?

Warwick et al (2018) point towards an emerging issue in design for wellbeing research: that of the blurring between participatory design as a means to create a design outcome which enhances wellbeing (e.g. a service, product or experience) and participatory design as a therapeutic process in itself. In this respect, the act of participating in a design process can promote feelings of wellbeing through for example, the co-designer feeling they are being listened to, and the social interaction that comes from taking part in these types of co-design activities.

Hong et al's (2018) contribution "Co-creating Happy Moments: A Case Study of Designing for People with Mental Health Challenges" builds on this theme further. They explored a participatory design approach to engage with and design meaningful experiences for two groups of mentally marginalised participants - a community in Norway that supports mental health and a group of youths in Finland. They used participatory design to enable the involvement of individuals and catalyse a positive change - though this change is observed rather than formally measured. This research raises some interesting questions around the blurring of boundaries between participatory design and art therapy. Interestingly, Hong et al (2018) also observed that they found their own involvement in the research as researchers also made them feel happy.

Continuing with the participatory design theme Garde et al (2018) in their paper titled "Design probes for people with dementia" developed a particular type of design probe to be used by people with dementia and reflected on the outcomes of using them as a source of inspiration for designers. Their aim with the probes was to "develop insight into the perspectives and life of people with dementia to enable a more empathic design approach and to identify needs, opportunities and ideas for mindful design interventions". Intriguingly, the authors found that more defined assignments 
(versus open-ended) had more value for designers as the defined assignments led to more sharing of personal details, and hence insights. They reflected that this contradicted other researchers' work who had found that the less instructive the probe tasks were, the more reflective and insightful the outcomes were. In terms of benefits to the participants, in the same way as both Warwick et al (2018) and Hong et al (2018), it was the act of participating which provided pleasurable benefits, allowing for personal reflection and enjoyment in doing the activities. Furthermore, the authors suggested that the participants felt empowered by using the probes because they felt they were helping with research

\subsection{Theme 2: New theories and frameworks}

Moving on to the second theme, where the final two contributions move into a more exploratory, theoretical space, we see new framings for designing meaningful and pleasurable experiences. Firstly, Legaard (2018) in their paper titled "The road to happiness is paved with playful intentions" start with the position that play activities, like meaningful experiences emerge when the activities of the experience comply with the interests, goals and passions of the person having the experience thus linking meaningfulness to intrinsic motivation. The paper provides an understanding of the components of a play experience through the introduction of an evolving 'Play Blueprint', which draws upon the theoretical foundations within play, experience design and positive design. Through this, the paper explores the relationship between meaningful and playful experiences, calling for designers to use the characteristics of play and playfulness to increase the intensity of an experience.

Finally, the contribution by Heekyoung and Stahl (2018) titled "Soma-Wearable Design: Integrating Somaesthetic Practice and Fashion design for Somatic Wellbeing" introduces a new concept - that of soma-wearable design for somatic wellbeing. As the authors explain, "Somaethetics is a branch of philosophy that grounds the meaning of human experience in the bodily presence". They propose that somaesthetic practice and fashion design have a meaningful overlap with their focus on body consciousness. Their paper describes how they conducted a design workshop with 14 fashion juniors to introduce the soma-wearable design approach and then observed how the approach was interpreted by the students. They point to some interesting future directions for the research, for example in investigating the link between somaesthetic reflection and self-perception and performance, e.g. through soma-wearables for mood change.

\section{Looking to the future}

Despite the SIGWELL theme containing a smaller number of accepted papers this year, the prevalence of participatory design as an underpinning methodological approach in the majority of the papers is noteworthy. However, perhaps one issue of debate, and of interest to explore further through discussion at the conference is the blurring of the boundaries between participatory design and art therapy. We see this emerging when it is the acts of involvement in participatory design which carry the therapeutic benefits and enhance subjective wellbeing, rather than the design outcome per se. In these situations, it is also interesting to reflect on how participation in designing for subjective wellbeing, can also have an emotional effect on the researchers involved - both positively through engagement in a meaningful experience, but also in raising more challenging questions of how designers are trained to work with people in sensitive scenarios around mental health.

Perhaps another challenge faced by researchers in the area of design for subjective wellbeing is that of scale. Many examples (both in this session, but also in the design for subjective wellbeing literature more generally) are individual case studies, relating to very specific populations, contexts and scenarios. From a methodical research perspective, the challenge lies in validating these approaches across other settings with other groups of people, so we start to build and converge on approaches for designing for subjective wellbeing supported by an evidence-base of their successes 
and failures. This challenge will be explored further through a SIGWELL workshop (Ozkaramanli et al 2018) also to be held at DRS2018, "Victories and Defeats: Current Practices in Design for Wellbeing".

\section{References}

Cain, R., Bitterman, N., Ludden, G., Mackrill, J., Ozcan, E., Petermans, A., Escobar-Tello, C. (2016) Introduction: Design for Health, Wellbeing and Happiness (SIGWELL), In P. Lloyd \& E. Bohemia, eds., Proceedings of DRS2016: Design + Research + Society - Future-Focused Thinking, Volume 4, pp 1434-1441. DOI: $10.21606 / d r s .2016 .625$

Garde, J.A., Van der Voort, M.C., Niedderer, K. (2018) Design Probes for people with dementia, In Proceedings of DRS2018: Catalyst, Limerick $25^{\text {th- }} 28^{\text {th }}$ June 2018

Heekyoung, A., Stahl, A., (2018) Soma-Wearable Design: Integrating Somaesthetic Practice and Fashion Design for Somatic Wellbeing, In Proceedings of DRS2018: Catalyst, Limerick $25^{\text {th }}-28^{\text {th }}$ June 2018

Hong, L., Zha, Y., Zhao, J. (2018) Co-creating Happy Moments: A Case Study of Designing for people with Mental Health Challenges, In Proceedings of DRS2018: Catalyst, Limerick $25^{\text {th }}-28^{\text {th }}$ June 2018

Legaard, J. (2018) The road to happiness is paved with playful intentions, In Proceedings of DRS2018: Catalyst, Limerick $25^{\text {th }}-28^{\text {th }}$ June 2018

Ozkaramanli, D., Petermans, A., Pohlmeyer, A. (2018) Victories and Defeats: Current Practices in Design for Wellbeing, Workshop at DRS2018, Limerick $25^{\text {th }}-28^{\text {th }}$ June 2018

Petermans, A. (2017). Design for Health, Wellbeing and Happiness Manifesto: Who, What, Why and How. Presented at the World Design Summit, Montreal, Canada, $17^{\text {th }}-19^{\text {th }}$ October 2017

Warwick, L., Tinning, A., Smith, N., Young, R. (2018) Co-designing wellbeing: the commonality of needs between co-designers and mental health service users, In Proceedings of DRS2018: Catalyst, Limerick $25^{\text {th }}$. $28^{\text {th }}$ June 2018

About the Authors:

Dr Rebecca Cain is Reader in Experience Design and Associate Dean for Enterprise at Loughborough Design School. She is a founding convenor and current board member of the Design Research Society Special Interest Group on Design for Wellbeing, Happiness, and Health and the Hon Secretary of the Design Research Society.

Dr Ann Petermans is Doctor Assistant at the Faculty of Architecture and Arts at Hasselt University in Belgium, and chair of the Design Research Society Special Interest Group on Design for Wellbeing, Happiness, and Health.

Dr Anna Pohlmeyer is Assistant Professor at the Department of Industrial Design at Delft University of Technology in the Netherlands, co-director of the Delft Institute of Positive Design, and board member of the Design Research Society Special Interest Group on Design for Wellbeing, Happiness, and Health.

Prof Pieter Desmet is Professor of Design for Experience at the Department of Industrial Design at Delft University of Technology in the Netherlands, co-director of the Delft Institute of Positive Design, and board member of the Design Research Society Special Interest Group on Design for Wellbeing, Happiness, and Health. Dr Deger Ozkaramanli is Lecturer in Industrial Design at the University of Liverpool, member of the Delft Institute of Positive Design, and board member of the Design Research Society Special Interest Group on Design for Wellbeing, Happiness, and Health. 